\title{
Unsteady Flows of a Generalized Fractional Burgers' Fluid between Two Side Walls Perpendicular to a Plate
}

\author{
Jianhong Kang, Yingke Liu, and Tongqiang Xia \\ Key Laboratory of Gas and Fire Control for Coal Mines, School of Safety Engineering, China University of Mining and Technology, \\ Xuzhou 221116, China \\ Correspondence should be addressed to Jianhong Kang; jhkang@cumt.edu.cn
}

Received 13 December 2014; Accepted 26 March 2015

Academic Editor: Pavel Kurasov

Copyright (C) 2015 Jianhong Kang et al. This is an open access article distributed under the Creative Commons Attribution License, which permits unrestricted use, distribution, and reproduction in any medium, provided the original work is properly cited.

\begin{abstract}
The unsteady flows of a generalized fractional Burgers' fluid between two side walls perpendicular to a plate are studied for the case of Rayleigh-Stokes' first and second problems. Exact solutions of the velocity fields are derived in terms of the generalized Mittag-Leffler function by using the double Fourier transform and discrete Laplace transform of sequential fractional derivatives. The solution for Rayleigh-Stokes' first problem is represented as the sum of the Newtonian solutions and the non-Newtonian contributions, based on which the solution for Rayleigh-Stokes' second problem is constructed by the Duhamel's principle. The solutions for generalized second-grade fluid, generalized Maxwell fluid, and generalized Oldroyd-B fluid performing the same motions appear as limiting cases of the present solutions. Furthermore, the influences of fractional parameters and material parameters on the unsteady flows are discussed by graphical illustrations.
\end{abstract}

\section{Introduction}

Basic understanding of the flows for non-Newtonian fluids are of great importance in a number of practical engineering applications, such as the extrusion of polymer fluids, exotic lubricant, animal bloods, heavy oils, and colloidal and suspension solutions [1]. The essential difference between non-Newtonian and Newtonian fluids is that the constitutive relation connecting stress and strain rate in Newtonian fluids is linear but in non-Newtonian fluids is nonlinear. In order to characterize this special property exhibited by non-Newtonian fluids many models have been proposed, among which the differential type and rate type models are especially interesting and acquired a special status $[2,3]$. There have been a growing body of researches on this topic in the literature [4-8]. As one of the rate type models, the Burgers' model which was firstly presented by Burgers [9] is a kind of viscoelastic models. Its mechanical analogy is a Maxwell model and a Vogit model connected in series. The Maxwell and Oldroyd-B fluids which are frequently used in the viscoelastic theory can be treated as the special cases of Burgers' fluid. So it is expected that the Burgers' model can better capture the complex rheological characteristics of many real fluids than other models. Until now, the Burgers' model has been successfully applied in many studies [10-15].

More recently, the fractional calculus has achieved much success in the description of complex dynamic system and is widely applied to many fields [16-18], especially to nonNewtonian fluids. The starting point of the fractional derivative model of non-Newtonian fluids is usually a classical differential equation which is modified by replacing the time derivative of an integer order by a fractional derivative. This generalization has been found to be very flexible and useful in describing the viscoelastic behavior [19-21]. So far there has been a great deal of references concerning non-Newtonian fluids with fractional derivative model [22-27].

The availability of exact solutions for non-Newtonian fluids is of significance because such solutions not only can explain the physics of some fundamental flows, but also can be used as a benchmark for complicated numerical codes that have been developed for much more complex flows. However, exact solutions for the unsteady flows of viscoelastic fluids are very rare and difficult to obtain due to the nonlinearity of their constitutive equations. When the fractional calculus approach is introduced in the constitutive equations, the solvability becomes more difficult even though the problems 
are one-dimensional in case of simple geometries such as single plate or disk. The literature survey indicates that the Rayleigh-Stokes' first and second problems for flows between two side walls perpendicular to a plate are two of few problems that can be analytically solved. Fetecau et al. [28] presented some exact solutions of this problem for a second grade fluid, which was then extended to a generalized second grade fluid with a fractional derivative model by Khan [29]. The similar problem for a Maxwell fluid was discussed by Hayat et al. [30] and was extended to a fractional generalized Maxwell fluid by Vieru et al. [31].

In this work, we study the unsteady flows of a Burgers' fluid between two side walls perpendicular to a plate with a fractional derivative model. The following two cases are studied: (i) the flow induced by the impulsive motion of the bottom plate (Rayleigh-Stokes' first problem) and (ii) the flow induced by the periodic oscillation of the bottom plate (Rayleigh-Stokes' second problem). The exact solutions for the two problems are obtained in terms of generalized MittagLeffler function by using integral transform technique.

\section{Governing Equations}

The momentum and continuity equations for an incompressible fluid are given by

$$
\begin{gathered}
\rho \frac{d \mathbf{V}}{d t}=-\nabla p+\nabla \cdot \mathbf{S}, \\
\nabla \cdot \mathbf{V}=0,
\end{gathered}
$$

where $\rho$ is the density of the fluid, $\mathbf{V}$ the velocity, $p$ the pressure, $\mathbf{S}$ the extra stress tensor, and $d / d t$ the material time derivative.

For an ordinary Burgers' fluid, the extra stress tensor $\mathbf{S}$ satisfies

$$
\left(1+\lambda_{1} \frac{\delta}{\delta t}+\lambda_{2} \frac{\delta^{2}}{\delta t^{2}}\right) \mathbf{S}=\mu\left(1+\lambda_{3} \frac{\delta}{\delta t}\right) \mathbf{A}_{1}
$$

where $\mu$ is the dynamic viscosity, $\mathbf{A}_{1}=\nabla \mathbf{V}+(\nabla \mathbf{V})^{T}$ is the first Rivlin-Ericksen tensor with $T$ as the transpose operation, $\lambda_{1}$ and $\lambda_{3}$ are relaxation and retardation times with the dimension of time, and $\lambda_{2}$ is a material parameter with the dimension of time square. The operator $\delta / \delta t$ is the upper convected time derivative defined by

$$
\begin{aligned}
& \frac{\delta \mathbf{S}}{\delta t}=\frac{\partial \mathbf{S}}{\partial t}+\mathbf{V} \cdot \nabla \mathbf{S}-\nabla \mathbf{V} \cdot \mathbf{S}-\mathbf{S} \cdot(\nabla \mathbf{V})^{T} \\
& \frac{\delta^{2} \mathbf{S}}{\delta t^{2}}=\frac{\delta}{\delta t}\left(\frac{\delta \mathbf{S}}{\delta t}\right) .
\end{aligned}
$$

We consider an incompressible Burgers' fluid occupying the space above an infinite flat plate and between two side walls perpendicular to this plate, as shown in Figure 1. The side walls are extended to infinity in the $x$ - and $y$-directions and are located at $z=0$ and $z=d$. The velocity and extra stress tensor of fluids under consideration should have the following forms:

$$
\mathbf{V}=[u(y, z, t), 0,0], \quad \mathbf{S}=\mathbf{S}(y, z, t)
$$

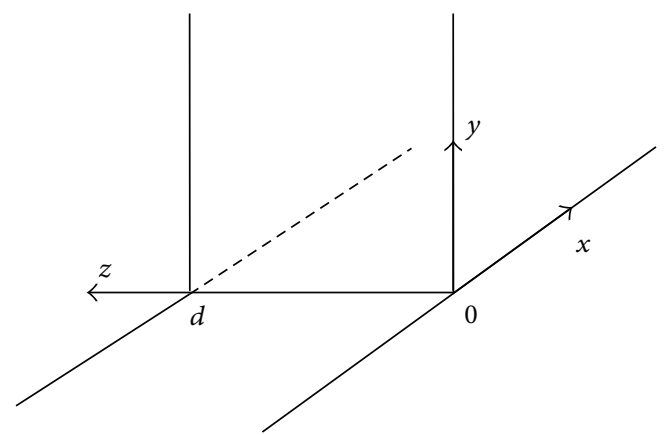

FIGURE 1: The schematic diagram of system considered here.

in the Cartesian coordinate system, where $u(y, z, t)$ is the velocity component in the $x$-direction.

According to (5), the continuity equation (2) is automatically satisfied and the constitutive equation (3) yields the following equations:

$$
\begin{gathered}
\left(1+\lambda_{1} \frac{\partial}{\partial t}+\lambda_{2} \frac{\partial^{2}}{\partial t^{2}}\right)\left[S_{y y}, S_{y z}, S_{z z}\right]=0 \\
\left(1+\lambda_{1} \frac{\partial}{\partial t}+\lambda_{2} \frac{\partial^{2}}{\partial t^{2}}\right) S_{x y}=\mu\left(1+\lambda_{3} \frac{\partial}{\partial t}\right) \frac{\partial u}{\partial y} \\
\left(1+\lambda_{1} \frac{\partial}{\partial t}+\lambda_{2} \frac{\partial^{2}}{\partial t^{2}}\right) S_{x z}=\mu\left(1+\lambda_{3} \frac{\partial}{\partial t}\right) \frac{\partial u}{\partial z}
\end{gathered}
$$

Assuming the fluids to be at rest initially, from (6) we can conclude that $S_{y y}=S_{y z}=S_{z z}=0$.

The governing equations corresponding to a generalized fractional Burgers' fluid performing the same motion can be obtained from (7) and (8) by substituting the time derivatives with fractional derivatives $[32,33]$, that is,

$$
\begin{aligned}
& \left(1+\lambda_{1}^{\alpha} \frac{\partial^{\alpha}}{\partial t^{\alpha}}+\lambda_{2}^{\alpha} \frac{\partial^{2 \alpha}}{\partial t^{2 \alpha}}\right) S_{x y}=\mu\left(1+\lambda_{3}^{\beta} \frac{\partial^{\beta}}{\partial t^{\beta}}\right) \frac{\partial u}{\partial y}, \\
& \left(1+\lambda_{1}^{\alpha} \frac{\partial^{\alpha}}{\partial t^{\alpha}}+\lambda_{2}^{\alpha} \frac{\partial^{2 \alpha}}{\partial t^{2 \alpha}}\right) S_{x z}=\mu\left(1+\lambda_{3}^{\beta} \frac{\partial^{\beta}}{\partial t^{\beta}}\right) \frac{\partial u}{\partial z}
\end{aligned}
$$

where $\partial^{\alpha} / \partial t^{\alpha}$ is the fractional derivative of order $\alpha$ with respect to $t$, which is defined as [34]

$$
\begin{array}{r}
\frac{\partial^{\alpha} f(t)}{\partial t^{\alpha}}=\frac{1}{\Gamma(k-\alpha)} \frac{d^{k}}{d t^{k}} \int_{0}^{t}(t-\tau)^{k-\alpha-1} f(\tau) d \tau, \\
(k-1 \leq \alpha<k),
\end{array}
$$

and $\Gamma(\cdot)$ is the Gamma function.

Then, from (1) together with (9) one can obtain the final governing equation for a generalized fractional Burgers' fluid in the absence of pressure gradient as follows:

$$
\left(1+\lambda_{1}^{\alpha} \frac{\partial^{\alpha}}{\partial t^{\alpha}}+\lambda_{2}^{\alpha} \frac{\partial^{2 \alpha}}{\partial t^{2 \alpha}}\right) \frac{\partial u}{\partial t}=v\left(1+\lambda_{3}^{\beta} \frac{\partial^{\beta}}{\partial t^{\beta}}\right)\left(\frac{\partial^{2} u}{\partial y^{2}}+\frac{\partial^{2} u}{\partial z^{2}}\right)
$$

where $\nu=\mu / \rho$ is the kinematic viscosity. 


\section{Exact Solutions of Unsteady Flows}

3.1. Flow Induced by the Impulsive Motion of the Plate. Initially, the fluid is at rest and then the plate is suddenly brought to a steady velocity $U_{0}$ at the moment $t=0^{+}$. Such a motion is termed as the Rayleigh-Stokes' first problem in the literature. In this case, the flow is governed by (11) and the initial-boundary conditions can be expressed as

$$
\begin{gathered}
u(y, z, 0)=0, \quad y>0,0 \leq z \leq d, \\
u(0, z, t)=U_{0} \quad 0<z<d, t>0, \\
u(y, 0, t)=u(y, d, t)=0, \quad y>0, t>0 .
\end{gathered}
$$

Introducing the dimensionless parameters

$$
u^{*}=\frac{u}{U_{0}}, \quad t^{*}=\frac{t}{d / U_{0}}, \quad\left(x^{*}, y^{*}, z^{*}\right)=\frac{(x, y, z)}{d},
$$

we can obtain the following dimensionless problem:

$$
\begin{gathered}
\left(1+\lambda_{1}^{*} \frac{\partial^{\alpha}}{\partial t^{* \alpha}}+\lambda_{2}^{*} \frac{\partial^{2 \alpha}}{\partial t^{* 2 \alpha}}\right) \frac{\partial u^{*}}{\partial t^{*}} \\
=\frac{1}{\operatorname{Re}}\left(1+\lambda_{3}^{*} \frac{\partial^{\beta}}{\partial t^{* \beta}}\right)\left(\frac{\partial^{2} u^{*}}{\partial y^{* 2}}+\frac{\partial^{2} u^{*}}{\partial z^{* 2}}\right), \\
u\left(y^{*}, z^{*}, 0\right)=0, \quad y^{*}>0,0 \leq z^{*} \leq 1, \\
u\left(0, z^{*}, t^{*}\right)=1, \quad 0<z^{*}<1, t^{*}>0, \\
u\left(y^{*}, 0, t^{*}\right)=u\left(y^{*}, 1, t^{*}\right)=0, \quad y^{*}>0, t^{*}>0,
\end{gathered}
$$

where $\lambda_{1}^{*}, \lambda_{2}^{*}$, and $\lambda_{3}^{*}$ and $\operatorname{Re}$ are the dimensionless relaxation time, material parameter, retardation time, and Reynolds number, respectively, defined as

$$
\begin{array}{ll}
\lambda_{1}^{*}=\lambda_{1}^{\alpha}\left(\frac{U_{0}}{d}\right)^{\alpha}, & \lambda_{2}^{*}=\lambda_{2}^{\alpha}\left(\frac{U_{0}}{d}\right)^{2 \alpha}, \\
\lambda_{3}^{*}=\lambda_{3}^{\beta}\left(\frac{U_{0}}{d}\right)^{\beta}, & \operatorname{Re}=\frac{U_{0} d}{v} .
\end{array}
$$

It should be noted that in order to solve a well-posed problem for (14) additional conditions apart from (15)-(17) are supposed to be attached, that is

$$
\begin{aligned}
\frac{\partial u^{*}\left(y^{*}, z^{*}, 0\right)}{\partial t^{*}}= & \frac{\partial^{2} u^{*}\left(y^{*}, z^{*}, 0\right)}{\partial t^{* 2}}=0, \\
& y^{*}>0, \quad 0 \leq z^{*} \leq 1, \\
u^{*}\left(y^{*}, z^{*}, t^{*}\right), & \frac{\partial u^{*}\left(y^{*}, z^{*}, t^{*}\right)}{\partial y^{*}} \longrightarrow 0 \\
& \text { as } y^{*} \longrightarrow \infty, \quad t^{*}>0 .
\end{aligned}
$$

The additional condition (19) is adopted for the derivation of analytical solution and has no explicit physical significance. However, without loss of generality, the adoption of such condition does not detract from overall conclusions for the comparison of flow behavior for different rheological models [27].

For the sake of brevity and convenience, we omit the asterisks "*” and keep the same notation for all variables from here on.

To solve the partial differential equation (14) subject to conditions (15)-(17), (19), and (20), the Fourier sine transform with respect to $y$ and the finite Fourier sine transform with respect to $z$ will be applied. The transform and its inversion are defined as

$$
\begin{gathered}
\bar{u}\left(\xi, \zeta_{n}, t\right)=\sqrt{\frac{2}{\pi}} \int_{0}^{\infty} \int_{0}^{1} u(y, z, t) \sin y \xi \sin z \zeta_{n} d y d z, \\
u(y, z, t)=2 \sqrt{\frac{2}{\pi}} \int_{0}^{\infty} \sum_{n=1}^{\infty} \bar{u}\left(\xi, \zeta_{n}, t\right) \sin y \xi \sin z \zeta_{n} d \xi
\end{gathered}
$$

in which $\zeta_{n}=n \pi$.

Taking the transform (21) to both sides of (14) and taking into account the initial-boundary conditions, one can find that

$$
\begin{aligned}
&\left(1+\lambda_{1} \frac{\partial^{\alpha}}{\partial t^{\alpha}}+\lambda_{2} \frac{\partial^{2 \alpha}}{\partial t^{2 \alpha}}\right) \frac{\partial \bar{u}}{\partial t} \\
&= \frac{1}{\operatorname{Re}}\left(1+\lambda_{3} \frac{\partial^{\beta}}{\partial t^{\beta}}\right) \\
& \cdot\left\{-\left(\xi^{2}+\zeta_{n}^{2}\right) \bar{u}+\sqrt{\frac{2}{\pi}} \frac{\xi}{\zeta_{n}}\left[1-(-1)^{n}\right]\right\}, \\
& \bar{u}\left(\xi, \zeta_{n}, 0\right)=\frac{\partial u\left(\xi, \zeta_{n}, 0\right)}{\partial t}=\frac{\partial^{2} \bar{u}\left(\xi, \zeta_{n}, 0\right)}{\partial t^{2}}=0 .
\end{aligned}
$$

To obtain an exact solution of (23) subject to the initial condition (24), the Laplace transform with respect to $t$ is further applied.

Let

$$
\widetilde{\bar{u}}\left(\xi, \zeta_{n}, s\right)=\int_{0}^{\infty} e^{-s t} \bar{u}\left(\xi, \zeta_{n}, t\right) d t
$$

be the Laplace transform image function of $\bar{u}\left(\xi, \zeta_{n}, t\right)$. By applying the Laplace transform, we arrive at

$$
\begin{aligned}
& \tilde{\bar{u}}\left(\xi, \zeta_{n}, s\right) \\
& =\sqrt{\frac{2}{\pi}} \frac{\xi}{\zeta_{n}} \frac{\left[1-(-1)^{n}\right] \operatorname{Re}^{-1} s^{-1}\left[1+\lambda_{3} \operatorname{sign}(1-\beta) s^{\beta}\right]}{\left[\lambda_{2} s^{2 \alpha+1}+\lambda_{1} s^{\alpha+1}+s+\operatorname{Re}^{-1}\left(\xi^{2}+\zeta_{n}^{2}\right)\left(1+\lambda_{3} s^{\beta}\right)\right]},
\end{aligned}
$$

where

$$
\operatorname{sign}(1-\beta)= \begin{cases}-1, & \beta>1 ; \\ 0, & \beta=1 ; \\ 1, & 0<\beta<1 .\end{cases}
$$


For a well presentation of the final results, (26) is rewritten as an equivalent form

$$
\begin{aligned}
\widetilde{\bar{u}}= & \sqrt{\frac{2}{\pi}}\left[1-(-1)^{n}\right] \frac{\xi}{\zeta_{n}\left(\xi^{2}+\zeta_{n}^{2}\right)}\left[\frac{1}{s}-\frac{1}{s+\operatorname{Re}^{-1}\left(\xi^{2}+\zeta_{n}^{2}\right)}\right] \\
& -\sqrt{\frac{2}{\pi}} \frac{\left[1-(-1)^{n}\right] \xi}{\zeta_{n}\left(\xi^{2}+\zeta_{n}^{2}\right)} \frac{1}{s+\operatorname{Re}^{-1}\left(\xi^{2}+\zeta_{n}^{2}\right)} \widetilde{H}\left(\xi, \zeta_{n}, s\right),
\end{aligned}
$$

where

$$
\begin{aligned}
\widetilde{H}\left(\xi, \zeta_{n}, s\right) & \\
= & \frac{\xi^{2}+\zeta_{n}^{2}}{\operatorname{Re}} \\
& \cdot \frac{\lambda_{2} s^{2 \alpha}+\lambda_{1} s^{\alpha}+\operatorname{Re}^{-1} \varepsilon \lambda_{3}\left(\xi^{2}+\zeta_{n}^{2}\right) s^{\beta-1}-\lambda_{3} \operatorname{sign}(1-\beta) s^{\beta}}{\left[\lambda_{2} s^{2 \alpha+1}+\lambda_{1} s^{\alpha+1}+s+\operatorname{Re}^{-1}\left(\xi^{2}+\zeta_{n}^{2}\right)\left(1+\lambda_{3} s^{\beta}\right)\right]}
\end{aligned}
$$

and $\varepsilon=1-\operatorname{sign}(1-\beta)$.

Taking the inverse Laplace transform to (28), we get that

$$
\begin{aligned}
\bar{u}\left(\xi, \zeta_{n}, t\right)= & \sqrt{\frac{2}{\pi}} \frac{\left[1-(-1)^{n}\right] \xi}{\zeta_{n}\left(\xi^{2}+\zeta_{n}^{2}\right)}\left[1-e^{-\left(\left(\xi^{2}+\zeta_{n}^{2}\right) / \mathrm{Re}\right) t}\right] \\
& -\sqrt{\frac{2}{\pi} \frac{\left[1-(-1)^{n}\right] \xi}{\zeta_{n}\left(\xi^{2}+\zeta_{n}^{2}\right)}} \\
& \cdot \int_{0}^{t} e^{-\left(\left(\xi^{2}+\zeta_{n}^{2}\right) / \operatorname{Re}\right)(t-\tau)} H\left(\xi, \zeta_{n}, \tau\right) d \tau
\end{aligned}
$$

in which $H\left(\xi, \zeta_{n}, t\right)$ is the inverse Laplace transform of $\widetilde{H}\left(\xi, \zeta_{n}, s\right)$. For a Burgers' fluid $\left(\lambda_{1} \neq 0\right.$ and $\left.\lambda_{2} \neq 0\right), H\left(\xi, \zeta_{n}, t\right)$ can be expressed as

$$
\begin{aligned}
H\left(\xi, \zeta_{n}, t\right)=\sum_{k=0}^{\infty} \sum_{l, j, m \geq 0}^{l+j+m=k} & (-1)^{k} \frac{\lambda_{3}^{j}\left[\operatorname{Re}^{-1}\left(\xi^{2}+\zeta_{n}^{2}\right)\right]^{k-m+1}}{\lambda_{2}^{k+1} l ! j ! m !} \\
\cdot\{ & \lambda_{1} t^{k \alpha-\varphi-2} E_{\alpha,-\varphi-1}^{(k)}\left(-\frac{\lambda_{1}}{\lambda_{2}} t^{\alpha}\right) \\
& +\lambda_{2} t^{k \alpha-\varphi-\alpha-2} E_{\alpha,-\varphi-\alpha-1}^{(k)}\left(-\frac{\lambda_{1}}{\lambda_{2}} t^{\alpha}\right) \\
& +\operatorname{Re}^{-1} \varepsilon \lambda_{3}\left(\xi^{2}+\zeta_{n}^{2}\right) t^{k \alpha+\alpha-\varphi-\beta-1} \\
& \times E_{\alpha, \alpha-\varphi-\beta}^{(k)}\left(-\frac{\lambda_{1}}{\lambda_{2}} t^{\alpha}\right) \\
& -\lambda_{3} \operatorname{sign}(1-\beta) t^{k \alpha+\alpha-\varphi-\beta-2} \\
& \left.\cdot E_{\alpha, \alpha-\varphi-\beta-1}^{(k)}\left(-\frac{\lambda_{1}}{\lambda_{2}} t^{\alpha}\right)\right\}
\end{aligned}
$$

where $\varphi=m+j \beta-k \alpha-\alpha-k-2$, and $E_{\alpha, \beta}=\sum_{k=0}^{\infty} t^{k} / \Gamma(k \alpha+\beta)$ denotes the generalized Mittag-Leffler function. In course of deriving (31), an important property of generalized MittagLeffler function is used as follows:

$$
\int_{0}^{\infty} e^{-s t} t^{k \alpha+\beta+1} E_{\alpha, \beta}^{(k)}\left( \pm c t^{\alpha}\right) d t=\frac{k ! s^{\alpha-\beta}}{\left(s^{\alpha} \mp c\right)^{k+1}} .
$$

Finally, inverting (30) by means of the inverse transform (22), we obtain the exact solution of the problem

$$
\begin{array}{r}
u(y, z, t)=\frac{8}{\pi} \sum_{n=1}^{\infty} \frac{\sin z \sigma_{n}}{\sigma_{n}} \int_{0}^{\infty} \frac{\xi \sin y \xi}{\xi^{2}+\sigma_{n}^{2}}\left[1-e^{-\left(\left(\xi^{2}+\sigma_{n}^{2}\right) / \mathrm{Re}\right) t}\right] d \xi \\
-\frac{8}{\pi} \sum_{n=1}^{\infty} \frac{\sin z \sigma_{n}}{\sigma_{n}} \int_{0}^{\infty} \frac{\xi \sin y \xi}{\xi^{2}+\sigma_{n}^{2}} \int_{0}^{t} e^{-\left(\left(\xi^{2}+\sigma_{n}^{2}\right) / \operatorname{Re}\right)(t-\tau)} \\
\cdot H\left(\xi, \sigma_{n}, \tau\right) d \tau d \xi,
\end{array}
$$

where $\sigma_{n}=(2 n-1) \pi$.

In view of the following formulae [27]:

$$
\begin{gathered}
\int_{0}^{\infty} \frac{\xi \sin y \xi}{\xi^{2}+a^{2}} d \xi=\frac{\pi}{2} e^{-a y} \\
\int_{0}^{\infty} \frac{\xi \sin y \xi}{\xi^{2}+b^{2}} e^{-a \xi^{2}} d \xi \\
=\frac{\pi}{4} e^{a b^{2}}\left[e^{-b y} \operatorname{erfc}\left(b \sqrt{a}-\frac{y}{2 \sqrt{a}}\right)\right. \\
\left.-e^{b y} \operatorname{erfc}\left(b \sqrt{a}+\frac{y}{2 \sqrt{a}}\right)\right],
\end{gathered}
$$

where $\operatorname{erfc}(\cdot)$ is the complementary error function, the solution (33) can be simplified as

$$
\begin{aligned}
& u(y, z, t) \\
& =u_{N}(x, y, y)-\frac{8}{\pi} \sum_{n=1}^{\infty} \frac{\sin z \sigma_{n}}{\sigma_{n}} \\
& \quad \cdot \int_{0}^{\infty} \frac{\xi \sin y \xi}{\xi^{2}+\sigma_{n}^{2}} \int_{0}^{t} e^{-\left(\left(\xi^{2}+\sigma_{n}^{2}\right) / \operatorname{Re}\right)(t-\tau)} H\left(\xi, \sigma_{n}, \tau\right) d \tau d \xi
\end{aligned}
$$

in which

$$
\begin{aligned}
& u_{N}(x, y, t) \\
& =4 \sum_{n=1}^{\infty} \frac{\sin z \sigma_{n}}{\sigma_{n}} e^{-\sigma_{n} y} \\
& -2 \sum_{n=1}^{\infty} \frac{\sin z \sigma_{n}}{\sigma_{n}}\left\{e^{-\sigma_{n} y} \operatorname{erfc}\left(\sigma_{n} \sqrt{\frac{t}{\operatorname{Re}}}-\frac{y}{2 \sqrt{t / \operatorname{Re}}}\right)\right. \\
& \left.\quad-e^{\sigma_{n} y} \operatorname{erfc}\left(\sigma_{n} \sqrt{\frac{t}{\operatorname{Re}}}+\frac{y}{2 \sqrt{t / \operatorname{Re}}}\right)\right\}
\end{aligned}
$$

is exactly the solution for a Newtonian fluid performing the same motion. 
It is easy to find that the velocity field $u(y, z, t)$ for a fractional Burgers' fluid given by (35) has two parts: the first part $u_{N}(x, y, t)$ corresponding to a Newtonian fluid performing the same motion and the second part on the right-hand side of (35) resulting from the viscoelastic property of a fractional Burgers' fluid.

Making $t \rightarrow \infty$ in (35), the steady velocity field for a fractional Burgers' fluid is obtained as follows:

$$
u_{s}(y, z, t)=4 \sum_{n=1}^{\infty} \frac{\sin z \sigma_{n}}{\sigma_{n}} e^{-\sigma_{n} y} .
$$

The steady volume flux corresponding to (37) is given by

$$
Q(\infty)=\int_{0}^{\infty} \int_{0}^{d} u(y, z, t) d z d y=\frac{8}{\pi^{3}} \sum_{n=1}^{\infty} \frac{1}{(2 n-1)^{3}} .
$$

In some limiting cases, the present solution for a generalized Burgers' fluid can be reduced to those corresponding to a generalized second-grade fluid, Maxwell fluid, and OldroydB fluid.

Remark 1. If one takes $\lambda_{1} \rightarrow 0$ and $\lambda_{2} \rightarrow 0$, (26) can be reduced to

$$
\begin{aligned}
\tilde{\bar{u}}\left(\xi, \sigma_{n}, s\right) \\
=\sqrt{\frac{2}{\pi}} \frac{\xi}{\operatorname{Re} \sigma_{n}} \frac{1+\lambda_{3} \operatorname{sign}(1-\beta) s^{\beta}}{s\left[s+\operatorname{Re}^{-1}\left(\xi^{2}+\sigma_{n}^{2}\right)\left(1+\lambda_{3} s^{\beta}\right)\right]} \\
=\sqrt{\frac{2}{\pi}} \frac{\xi}{\operatorname{Re} \sigma_{n}} \sum_{k=0}^{\infty}(-1)^{k}\left[\operatorname{Re}^{-1}\left(\xi^{2}+\sigma_{n}^{2}\right)\right]^{k} \\
\cdot \frac{s^{-k \beta-\beta-1}+\operatorname{Re}^{-1} \lambda_{3} s^{k \beta-1}}{\left[s^{1-\beta}+\operatorname{Re}^{-1}\left(\xi^{2}+\sigma_{n}^{2}\right)\right]^{k+1}} .
\end{aligned}
$$

Applying the inverse Laplace transform term by term on (39) and then using the formulae (22), we arrive at

$$
\begin{aligned}
& u(y, z, t) \\
& =\frac{8}{\pi} \sum_{n=1}^{\infty} \frac{\sin z \sigma_{n}}{\sigma_{n}} \\
& \cdot \int_{0}^{\infty} \frac{\xi \sin y \xi}{\xi^{2}+\sigma_{n}^{2}} \sum_{k=0}^{\infty} \frac{(-1)^{k}\left[\operatorname{Re}^{-1}\left(\xi^{2}+\sigma_{n}^{2}\right)\right]^{k+1}}{k !} \\
& \quad \times\left\{t^{k+1} E_{1-\beta, k \beta+2}^{(k)}\left(-\frac{\xi^{2}+\sigma_{n}^{2}}{\operatorname{Re}} t^{1-\beta}\right)\right. \\
& \quad+\operatorname{Re}^{-1} \operatorname{sign}(1-\beta) \\
& \left.\quad \times t^{k-\beta+1} E_{1-\beta, k \beta-\beta+2}^{(k)}\left(-\frac{\xi^{2}+\sigma_{n}^{2}}{\operatorname{Re}} t^{1-\beta}\right)\right\} d \xi
\end{aligned}
$$

which is an equivalent form of the solution for a generalized second-grade fluid obtained by Khan and Wang [29].
Remark 2. If one takes $\lambda_{2} \rightarrow 0$ and $\lambda_{3} \rightarrow 0$, (26) can be reduced to

$$
\begin{aligned}
& \tilde{\bar{u}}\left(\xi, \sigma_{n}, s\right) \\
& =\sqrt{\frac{2}{\pi}} \frac{\xi}{\operatorname{Re} \sigma_{n}} \frac{1}{s\left[\lambda_{1} s^{\alpha+1}+s+\operatorname{Re}^{-1}\left(\xi^{2}+\sigma_{n}^{2}\right)\right]} \\
& \quad=\sqrt{\frac{2}{\pi}} \frac{\xi}{\sigma_{n}} \sum_{k=0}^{\infty} \frac{(-1)^{k}\left[\operatorname{Re}^{-1}\left(\xi^{2}+\sigma_{n}^{2}\right)\right]^{k}}{\operatorname{Re} \lambda_{1}^{k+1}} \frac{s^{-k-2}}{\left(s^{\alpha}+1 / \lambda_{1}\right)^{k+1}} .
\end{aligned}
$$

Applying the inverse Laplace transform term by term on (41) and then using the formulae (22), we arrive at

$$
\begin{aligned}
& u(y, z, t) \\
& =\frac{8}{\pi} \sum_{n=1}^{\infty} \frac{\sin z \sigma_{n}}{\sigma_{n}} \\
& \quad \int_{0}^{\infty} \frac{\xi \sin y \xi}{\xi^{2}+\sigma_{n}^{2}} \sum_{k=0}^{\infty}\left\{\frac{(-1)^{k}\left[\operatorname{Re}^{-1}\left(\xi^{2}+\sigma_{n}^{2}\right)\right]^{k+1}}{\lambda_{1}^{k+1} k !}\right. \\
& \left.\times t^{k \alpha+\alpha+k+1} E_{\alpha, k+\alpha+2}^{(k)}\left(-\frac{t^{\alpha}}{\lambda_{1}}\right)\right\} d \xi
\end{aligned}
$$

which is exactly the velocity field for a generalized Maxwell fluid obtained by Vieru et al. [31].

Remark 3. If one takes $\lambda_{2} \rightarrow 0$ in (29), the solution for a generalized Oldroyd-B fluid performing the same motion is recovered as follows:

$$
\begin{aligned}
& u(y, z, t) \\
& =u_{N}(x, y, y)-\frac{8}{\pi} \sum_{n=1}^{\infty} \frac{\sin z \sigma_{n}}{\sigma_{n}} \\
& \quad \cdot \int_{0}^{\infty} \frac{\xi \sin y \xi}{\xi^{2}+\sigma_{n}^{2}} \times \int_{0}^{t} e^{-\left(\left(\xi^{2}+\sigma_{n}^{2}\right) / \operatorname{Re}\right)(t-\tau)} G\left(\xi, \sigma_{n}, \tau\right) d \tau d \xi,
\end{aligned}
$$

where

$$
\begin{aligned}
G\left(\xi, \sigma_{n}, t\right) \\
=\sum_{k=0}^{\infty} \sum_{m=0}^{k}(-1)^{k} \frac{\left[\operatorname{Re}^{-1}\left(\xi^{2}+\sigma_{n}^{2}\right)\right]^{k-m+1}}{\lambda_{1}^{k+1} m !(k-m) !} \\
\quad \cdot\left\{\lambda_{1} t^{\phi} E_{\theta+1, \gamma}^{(k)}\left(-\frac{\lambda_{3}\left(\xi^{2}+\sigma_{n}^{2}\right)}{\lambda_{1} \operatorname{Re}} t^{\alpha}\right)\right.
\end{aligned}
$$




$$
\begin{aligned}
& -\lambda_{3} \operatorname{sign}(1-\beta) t^{\phi+\theta} E_{\theta+1, \theta+\gamma}^{(k)}\left(-\frac{\lambda_{3}\left(\xi^{2}+\sigma_{n}^{2}\right)}{\lambda_{1} \operatorname{Re}} t^{\alpha}\right) \\
& +\operatorname{Re}^{-1} \varepsilon \lambda_{3}\left(\xi^{2}+\sigma_{n}^{2}\right) t^{\phi+\theta+1} \\
& \left.\cdot E_{\theta+1, \theta+\gamma+1}^{(k)}\left(-\frac{\lambda_{3}\left(\xi^{2}+\sigma_{n}^{2}\right)}{\lambda_{1} \operatorname{Re}} t^{\alpha}\right)\right\}
\end{aligned}
$$

with $\phi=k \alpha+k-m,=k \beta-m+1$, and $\theta=\alpha-\beta$.

3.2. Flow Induced by General Periodic Oscillations of the Plate. Consider the flow is caused by the plate whose velocity is of the form $U_{0} f(t)$. Here, $U_{0}$ is a constant and $f(t)$ is a general periodic oscillation with period $T_{0}$. Such a motion is termed as the Rayleigh-Stokes' second problem in the literature. In this case, by introducing the same dimensionless parameters as in (13) and dropping the asterisks, the dimensionless governing equation is still (14) and the dimensionless boundary conditions are expressed as

$$
\begin{gathered}
u(0, z, t)=f(t), \quad 0<z<1, \\
u(y, 0, t)=u(y, 1, t)=0, \quad y>0, \\
u(y, z, t), \quad \frac{\partial u(y, z, t)}{\partial y} \longrightarrow \infty, \quad \text { as } y \longrightarrow \infty .
\end{gathered}
$$

Based on the result obtained in Section 3.1, the solution for the present problem can be given by the so called Duhamel's principle [35]:

$u(y, z, t)=\int_{0}^{t} u^{(1)}(y, z, t-\tau) f^{\prime}(\tau) d \tau+f(0) u^{(1)}(y, z, t)$,

where for convenience $u^{(1)}(y, z, t)$ denotes the solution of the first problem given by (35). However, due to the cumbersome calculation of integral (46), we use another technique to handle this problem and the result is supposed to be more concise than that of (46).

First, $f(t)$ is expanded as the complex Fourier series

$$
f(t)=\sum_{k=-\infty}^{\infty} a_{k} e^{i k \omega_{0} t},
$$

where $\omega_{0}=\left(2 \pi / T_{0}\right) \cdot\left(d / U_{0}\right)$ is the nonzero dimensionless fundamental frequency and the coefficient $a_{k}$ can be calculated as

$$
a_{k}=\frac{d}{U_{0} T_{0}} \int_{0}^{T_{0} U_{0} / d} f(t) e^{-i k \omega_{0} t} d t .
$$

Then, we attempt to find the solution by means of the temporal Fourier transform. The transform and its inverse transform are defined by

$$
\begin{gathered}
\widehat{u}(y, z, \omega)=\int_{-\infty}^{\infty} e^{-i \omega t} u(y, z, t) d t, \\
u(y, z, t)=\frac{1}{2 \pi} \int_{-\infty}^{\infty} e^{i \omega t} \widehat{u}(y, z, \omega) d \omega .
\end{gathered}
$$

Having in mind (47) and taking the transform (49) to (14) subject to the boundary conditions (45), we arrive at

$$
\begin{gathered}
\left(\frac{\partial^{2}}{\partial y^{2}}+\frac{\partial^{2}}{\partial z^{2}}-c^{2}\right) \widehat{u}(x, y, \omega)=0, \\
\widehat{u}(y, 0, \omega)=\widehat{u}(y, 1, \omega)=0, \quad y>0, \\
\widehat{u}(y, z, w), \quad \frac{\widehat{u}(y, z, \omega)}{\partial y} \longrightarrow 0, \quad \text { as } y \longrightarrow \infty, \\
\widehat{u}(0, z, \omega)=2 \pi \sum_{-\infty}^{\infty} a_{k} \delta\left(\omega-k \omega_{0}\right), \quad 0<z<1,
\end{gathered}
$$

where $\delta(\cdot)$ is the Dirac delta function and

$$
c^{2}=\frac{i \omega\left[1+\lambda_{1}(i \omega)^{\alpha}+\lambda_{2}(i \omega)^{2 \alpha}\right]}{\operatorname{Re}^{-1}\left[1+\lambda_{3}(i \omega)^{\beta}\right]}
$$

with $(i \omega)^{\alpha}=|\omega|^{\alpha}[\cos (\pi \alpha / 2)+i \operatorname{sign} \omega \sin (\pi \alpha / 2)]$.

In course of obtaining (51) and (54), the following Fourier transform formulae of fractional derivative and Dirac delta function are used:

$$
\begin{gathered}
\int_{-\infty}^{\infty} \frac{\partial^{\alpha} u(y, z, t)}{\partial t^{\alpha}} e^{-i \omega t} d t=(i \omega)^{\alpha} \widehat{u}(y, z, \omega), \\
\int_{-\infty}^{\infty} e^{-i\left(\omega-\omega_{0}\right) t} d t=2 \pi \delta\left(\omega-\omega_{0}\right) .
\end{gathered}
$$

Using the transform pair (21) and (22) again, from (51)(54) we can get that

$$
\widehat{u}(y, z, \omega)=8 \pi \sum_{n=1}^{\infty} \sum_{k=-\infty}^{\infty} \delta\left(\omega-k \omega_{0}\right) \frac{a_{k} \sin z \sigma_{n}}{\sigma_{n}} e^{-y \sqrt{\sigma_{n}^{2}+c^{2}}} .
$$

Finally, inverting (57) by using the inverse transform (50), we arrive at

$$
u(y, z, t)=4 \sum_{n=1}^{\infty} \sum_{k=-\infty}^{\infty} a_{k} \frac{\sin z \sigma_{n}}{\sigma_{n}} e^{i k \omega_{0} t-y \sqrt{\sigma_{n}^{2}+c_{k}^{2}}}
$$

with $c_{k}^{2}=\left.c^{2}\right|_{\omega=k \omega_{0}}$.

Equation (58) gives the complete analytic solution of the velocity field due to the general periodic oscillation of the plate. As three special cases of the oscillation, we consider $f(t)$ in the following form:

(1) $f(t)=e^{i \omega_{0} t}, \quad a_{1}=0, a_{k}=0(k \neq 1)$

(2) $f(t)=\cos \omega_{0} t, \quad a_{1}=a_{-1}=\frac{1}{2}, a_{k}=0$, otherwise

(3) $f(t)=\sin \omega_{0} t, \quad a_{1}=a_{-1}=\frac{1}{2 i}, a_{k}=0$, otherwise. 
After substituting the corresponding Fourier coefficients of (59) into (58), the solutions can be easily obtained as follows:

$$
\begin{aligned}
& u_{1}=4 \sum_{n=1}^{\infty} \frac{\sin z \sigma_{n}}{\sigma_{n}} e^{i \omega_{0} t-y \sqrt{\sigma_{n}^{2}+c_{1}^{2}}}, \\
& u_{2}=2 \sum_{n=1}^{\infty} \frac{\sin z \sigma_{n}}{\sigma_{n}}\left[e^{i \omega_{0} t-y \sqrt{\sigma_{n}^{2}+c_{1}^{2}}}+e^{-i \omega_{0} t-y \sqrt{\sigma_{n}^{2}+c_{-1}^{2}}}\right], \\
& u_{2}=-2 i \sum_{n=1}^{\infty} \frac{\sin z \sigma_{n}}{\sigma_{n}}\left[e^{i \omega_{0} t-y \sqrt{\sigma_{n}^{2}+c_{1}^{2}}}+e^{-i \omega_{0} t-y \sqrt{\sigma_{n}^{2}+c_{-1}^{2}}}\right] .
\end{aligned}
$$

By letting $\lambda_{1}=\lambda_{2}=\lambda_{3}=0$ in (58) the exact solution for a Newtonian fluid performing the same motion can be recovered as follows:

$$
u_{N}(y, z, t)=4 \sum_{n=1}^{\infty} \sum_{k=-\infty}^{\infty} a_{k} \frac{\sin z \sigma_{n}}{\sigma_{n}} e^{i k \omega_{0} t-y \sqrt{\sigma_{n}^{2}-i k \omega_{0} \mathrm{Re}}} .
$$

Furthermore, the solutions for a generalized secondgrade fluid, Maxwell fluid, and Oldroyd-B fluid performing the same motion can be retrieved by taking corresponding limiting cases of the parameters $\lambda_{1}, \lambda_{2}$, and $\lambda_{3}$ in (55) and (58).

\section{Numerical Result and Discussion}

In this section, we plot the velocity fields according to the exact solutions obtained in the last section. For clarity, the symbols $u_{1}(y, z, t)$ and $u_{2}(y, z, t)$ are used to denote the solutions given by (33) and (60) in the following discussion, respectively.

The influences of the fractional parameters $\alpha$ and $\beta$ on the velocity field $u_{1}$ are illustrated in Figures 2 and 3 . It is shown in Figure 2 that $u_{1}$ decreases with the increase of $\alpha$, implying the suppressing effect of the parameter $\alpha$ on the fluid motion. On the contrary, the parameter $\beta$ plays a promoting role on the fluid motion as shown in Figure 3. The parameter $\alpha$ in the generalized Burgers' model is related with the relaxation term which characterizes the elasticity of fluid, while the parameter $\beta$ is related with the retardation term which characterizes the viscous damping of fluid. When $\alpha$ and $\beta$ tend to vanish, the Burgers' fluid reduces to a Newtonian fluid. Accordingly, the increase of $\alpha$ is indicative of the transition of a fluid phase to an elastic solid phase, and the increase of $\beta$ is to the contrary. Therefore, the results obtained in Figures 2 and 3 can be explained by the fact that the elasticity of fluid tends to reduce the velocity induced by the impulsive motion of a plate, while the viscous damping of fluid tends to enhance the velocity induced by the impulsive motion of a plate.

The effect of the material parameter $\lambda_{2}$ which distinguishes the Burgers' model from the Oldroyd-B model and Maxwell model is illustrated in Figure 4. It can be seen that the larger the value of $\lambda_{2}$ is, the smaller the velocity becomes, manifesting that $\lambda_{2}$ can reduce the fluid motion.

The Reynolds number Re is an important dimensionless parameter defining the laminar or turbulent flow. It is well known that the thickness of boundary layer is inversely

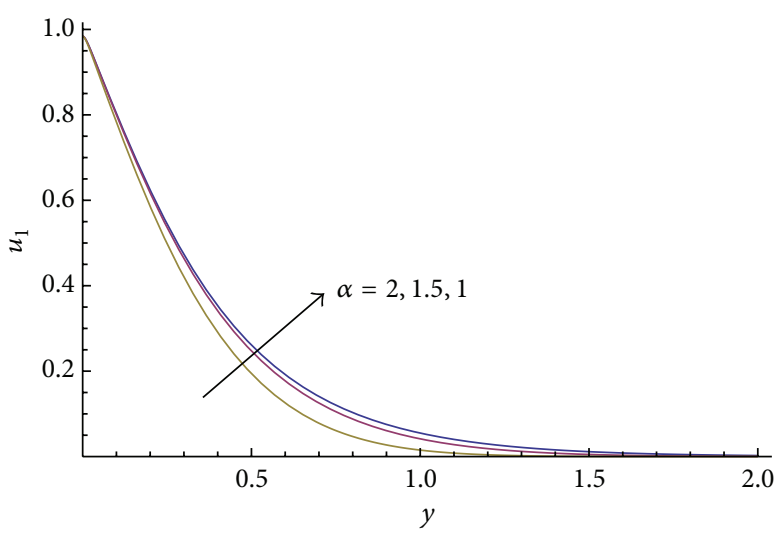

FIGURE 2: The influence of the fractional-order parameter $\alpha$ on the velocity profile $u_{1}(y, z, t)$ with $z=0.5, t=0.3, \beta=0.8, \operatorname{Re}=1$, $\lambda_{1}=5, \lambda_{2}=2$, and $\lambda_{3}=1$.

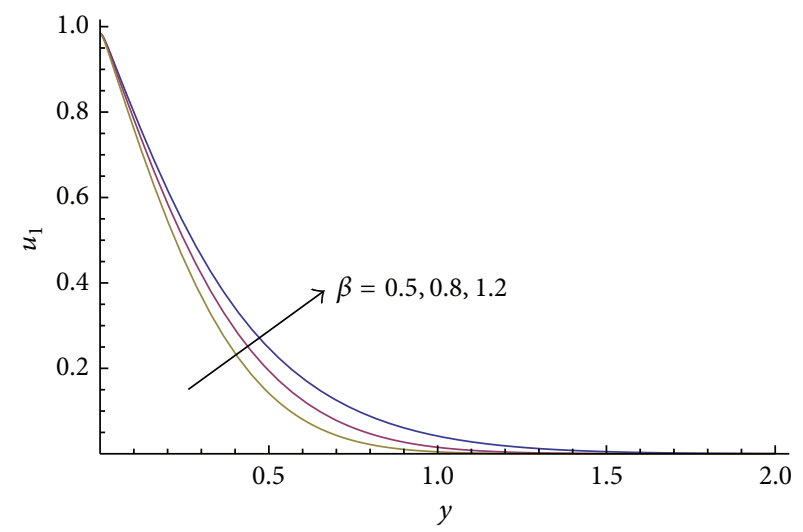

FIgURE 3: The influence of the fractional-order parameter $\beta$ on the velocity profile $u_{1}(y, z, t)$ with $z=0.5, t=0.3, \alpha=2, \operatorname{Re}=1, \lambda_{1}=5$, $\lambda_{2}=2$, and $\lambda_{3}=1$.

proportional to the value of Re. The present result shown in Figure 5 is also in agreement with this law. The Reynolds number $\mathrm{Re}$ can be taken as the ratio of inertial forces to viscous forces and consequently quantifies the relative importance of these two types of forces for given flow conditions. With the decrease of Re, the effect of viscous forces becomes dominant so that the vortices of flow can diffuse far away from the bottom plate.

Figure 6 explicitly displays the variations of velocity profile $u_{2}$ with $z$ at different time $t$. It is interestingly observed that at the moment $t=2.5$ the largest velocity value is not located in the middle of the two side walls but appears symmetrically near the two side walls. This physical phenomenon may result from the competing effects between the elasticity and viscous damping of viscoelastic fluid.

\section{Conclusions}

The objective of this paper is to provide exact solutions of unsteady flows for a generalized fractional Burgers' fluid between two side walls perpendicular to a plate. The unsteady 


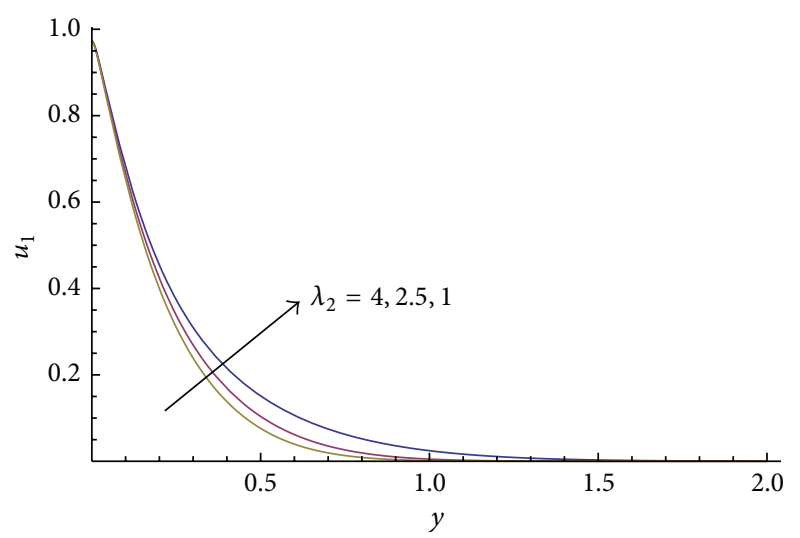

FIgURE 4: The influence of the material parameter $\lambda_{2}$ on the velocity profile $u_{1}(y, z, t)$ with $z=0.5, t=0.1, \alpha=2.5, \beta=0.5, \operatorname{Re}=1$, $\lambda_{1}=5$, and $\lambda_{3}=1$.

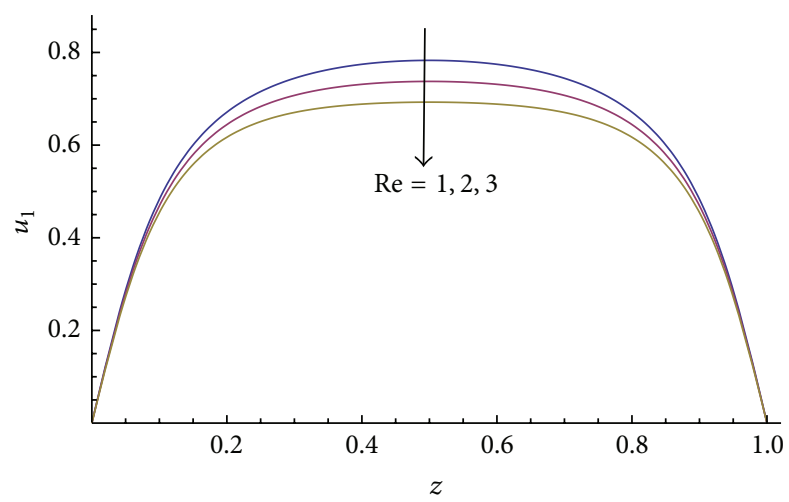

FIgURE 5: The influence of the Reynolds number Re on the velocity profile $u_{1}(y, z, t)$ with $y=0.1, t=0.3, \alpha=2, \beta=0.8, \lambda_{1}=5$, $\lambda_{2}=2$, and $\lambda_{3}=1$.

flows are induced by the impulsive motion or general periodic oscillations of the plate, which are, respectively, termed as the Rayleigh-Stokes' first and second problems. The analytic solutions of the two problems are obtained by using Fourier sine and Laplace transform methods in terms of MittagLeffler function. Moreover, the effects of various parameters are analyzed by plotting the velocity profiles according to the exaction solutions. The fractional constitutive model is more flexible and useful than the convectional model for characterizing the property of viscoelastic fluids, so it is expected that the present results will be of significance to fundamental research and practical applications in this field.

\section{Conflict of Interests}

The authors declare that there is no conflict of interests regarding the publication of this paper.

\section{Acknowledgments}

This work was supported by the Fundamental Research Funds for the Central Universities (Grant nos. 2014XT02

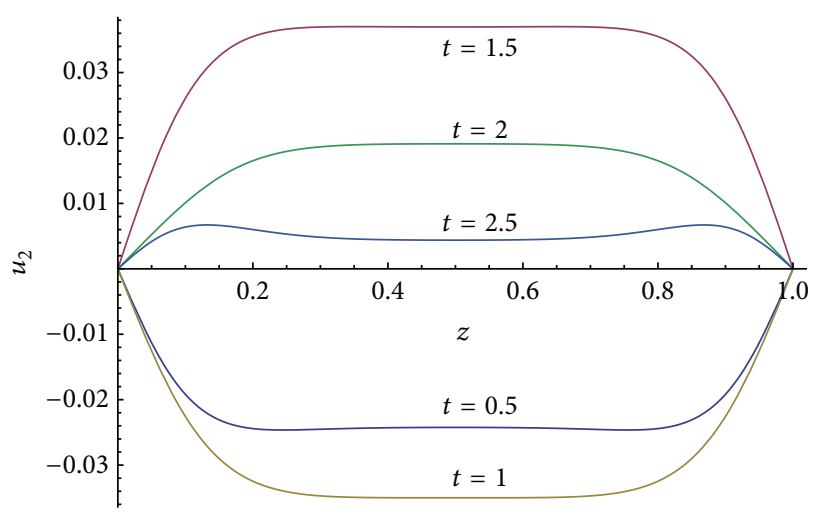

FIGURE 6: The variation of velocity profile $u_{2}(x, y, t)$ with $z$ for different time $t$ in which $\omega=5, \alpha=1.5, \beta=0.5, \lambda_{1}=8, \lambda_{2}=2$, $\lambda_{3}=1, \operatorname{Re}=1$, and $y=0.5$.

and 2014ZDPY03), the National Natural Science Foundation of China (Grant no. 11402293), the China Postdoctoral Science Foundation (Grant no. 2014M560458), the Program for Changjiang Scholars and Innovative Research Team in University (Grant no. IRT13098), A Program Funded by the Priority Academic Program Development of Jiangsu Higher Education Institutions, The Team Project Funded by 2014 Jiangsu Innovation and Entrepreneurship Program, and the Qing Lan Project of Jiangsu Province.

\section{References}

[1] K. R. Rajagopal, "Mechanics of non-Newtonian fluids," in Recent Developments in Theoretical Fluid Mechanics, vol. 291 of Pitmau Research Notes in Mathematics Series, pp. 129-162, Longman, 1993.

[2] J. E. Dunn and K. R. Rajagopal, "Fluid of differential type: critical review and thermodynamic analysis," International Journal of Non-Linear Mechanics, vol. 30, pp. 817-839, 1995.

[3] K. R. Rajagopal and A. R. Srinivasa, "A thermodynamic frame work for rate type fluid models," Journal of Non-Newtonian Fluid Mechanics, vol. 88, no. 3, pp. 207-227, 2000.

[4] C. Fetecau and J. Zierep, "The Rayleigh-Stokes-problem for a Maxwell fluid," Zeitschrift für Angewandte Mathematik und Physik, vol. 54, no. 6, pp. 1086-1093, 2003.

[5] M. E. Erdoğan and C. E. Imrak, "Effects of the side walls on unsteady flow of a second grade fluid over a plane wall," International Journal of Non-Linear Mechanics, vol. 43, no. 8, pp. 779-782, 2008.

[6] J. Kang, C. Fu, and W. Tan, "Thermal convective instability of viscoelastic fluids in a rotating porous layer heated from below," Journal of Non-Newtonian Fluid Mechanics, vol. 166, no. 1-2, pp. 93-101, 2011.

[7] M. S. Abel, J. V. Tawade, and J. N. Shinde, "The effects of MHD flow and heat transfer for the UCM fluid over a stretching surface in presence of thermal radiation," Advances in Mathematical Physics, vol. 2012, Article ID 702681, 21 pages, 2012.

[8] J. Kang, J. Niu, C. Fu, and W. Tan, "Coriolis effect on thermal convective instability of viscoelastic fluids in a rotating porous cylindrical annulus," Transport in Porous Media, vol. 98, no. 2, pp. 349-362, 2013. 
[9] J. M. Burgers, "Mechanical considerations-model system phenomenological theories of relaxation of viscosity," in First Report on Viscosity and Plasticity, J. M. Burgers, Ed., Nordemann Publishing Company, New York, NY, USA, 1935.

[10] T. Hayat, S. B. Khan, and M. Khan, "Exact solution for rotating flows of a generalized Burgers' fluid in a porous space," Applied Mathematical Modelling, vol. 32, no. 5, pp. 749-760, 2008.

[11] P. Ravindran, J. M. Krishnan, and K. R. Rajagopal, "A note on the flow of a Burgers' fluid in an orthogonal rheometer," International Journal of Engineering Science, vol. 42, no. 19-20, pp. 1973-1985, 2004.

[12] K.-X. Hu, J. Peng, and K.-Q. Zhu, "The linear stability of plane Poiseuille flow of Burgers fluid at very low Reynolds numbers," Journal of Non-Newtonian Fluid Mechanics, vol. 167-168, pp. 8794, 2012.

[13] R. Quintanilla and K. R. Rajagopal, "Further mathematical results concerning Burgers fluids and their generalizations," Zeitschrift für angewandte Mathematik und Physik, vol. 63, no. 1, pp. 191-202, 2012.

[14] I. Khan, F. Ali, and S. Shafie, "Stokes' second problem for magnetohydrodynamics flow in a Burgers' fluid: the cases $\gamma=$ $\lambda 2 / 4$ and $\gamma>\lambda 2 / 4$," PLoS ONE, vol. 8, no. 5, Article ID e61531, 2013.

[15] M. Jamil and C. Fetecau, "Starting solutions for the motion of a generalized Burgers' fluid between coaxial cylinders," Boundary Value Problems, vol. 2012, article 14, 2012.

[16] M. Xu and W. Tan, "Intermediate processes and critical phenomena: theory, method and progress of fractional operators and their applications to modern mechanics," Science in China, Series G: Physics Astronomy, vol. 49, no. 3, pp. 257-272, 2006.

[17] W. Zhong, C. Li, and J. Kou, "Numerical fractional-calculus model for two-phase flow in fractured media," Advances in Mathematical Physics, vol. 2013, Article ID 429835, 7 pages, 2013.

[18] D. Baleanu, H. M. Srivastava, V. Daftardar-Gejji, C. Li, and J. A. T. Machado, "Advanced topics in fractional dynamics," Advances in Mathematical Physics, vol. 2013, Article ID 723496, 1 page, 2013.

[19] C. Friedrich, "Relaxation and retardation functions of the Maxwell model with fractional derivatives," Rheologica Acta, vol. 30, no. 2, pp. 151-158, 1991.

[20] E. Bazhlekova and I. Bazhlekov, "Viscoelastic flows with fractional derivative models: computational approach by convolutional calculus of Dimovski," Fractional Calculus and Applied Analysis, vol. 17, no. 4, pp. 954-976, 2014.

[21] J.-S. Duan and X. Qiu, “The periodic solution of Stokes' second problem for viscoelastic fluids as characterized by a fractional constitutive equation," Journal of Non-Newtonian Fluid Mechanics, vol. 205, pp. 11-15, 2014.

[22] F. Ali, M. Norzieha, S. Sharidan, I. Khan, and T. Hayat, "New exact solutions of Stokes' second problem for an MHD second grade fluid in a porous space," International Journal of NonLinear Mechanics, vol. 47, no. 5, pp. 521-525, 2012.

[23] J. Kang and M. Y. Xu, "An exact solution for flow past an accelerated horizontal plate in a rotating fluid with the generalized Oldroyd-B model," Acta Mechanica Sinica, vol. 25, no. 4, pp. 463-469, 2009.

[24] J. Kang and M. Xu, "Exact solutions for unsteady unidirectional flows of a generalized second-order fluid through a rectangular conduit," Acta Mechanica Sinica, vol. 25, no. 2, pp. 181-186, 2009.

[25] M. Khan, "Partial slip effects on the oscillatory flows of a fractional Jeffrey fluid in a porous medium," Journal of Porous Media, vol. 10, no. 5, pp. 473-487, 2007.
[26] T. Hayat, M. Khan, and S. Asghar, "On the MHD flow of fractional generalized Burgers' fluid with modified Darcy's law," Acta Mechanica Sinica, vol. 23, no. 3, pp. 257-261, 2007.

[27] C. Fetecau, M. Jamil, and D. Vieru, “The Rayleigh-Stokes problem for an edge in a generalized Oldroyd-B fluid," Zeitschrift für angewandte Mathematik und Physik, vol. 60, no. 5, pp. 921-933, 2009.

[28] C. Fetecau, T. Hayat, and N. Ali, "Unsteady flow of a second grade fluid between two side walls perpendicular to a plate," Nonlinear Analysis. Real World Applications, vol. 9, no. 3, pp. 1236-1252, 2008.

[29] M. Khan and S. Wang, "Flow of a generalized second-grade fluid between two side walls perpendicular to a plate with a fractional derivative model," Nonlinear Analysis: Real World Applications, vol. 10, no. 1, pp. 203-208, 2009.

[30] T. Hayat, C. Fetecau, Z. Abbas, and N. Ali, "Flow of a Maxwell fluid between two side walls due to a suddenly moved plate," Nonlinear Analysis: Real World Applications, vol. 9, no. 5, pp. 2288-2295, 2008.

[31] D. Vieru, C. Fetecau, and C. Fetecau, "Flow of a viscoelastic fluid with the fractional Maxwell model between two side walls perpendicular to a plate," Applied Mathematics and Computation, vol. 200, no. 1, pp. 459-464, 2008.

[32] M. Jamil, N. A. Khan, and A. A. Zafar, "Translation flows of an Oldroyd-B fluid with fractional derivatives," Computers \& Mathematics with Applications, vol. 62, no. 3, pp. 1540-1553, 2011.

[33] M. Khan, A. Anjum, C. Fetecau, and H. Qi, "Exact solutions for some oscillating motions of a fractional Burgers' fluid," Mathematical and Computer Modelling, vol. 51, no. 5-6, pp. 682692, 2010.

[34] I. Podlubny, Fractional Differential Equations, Academic Press, San Diego, Calif, USA, 1999.

[35] C. S. Yih, Fluid Mechanics: A Concise Introduction to the Theory, West River Press, Ann Arbor, Mich, USA, 1977. 


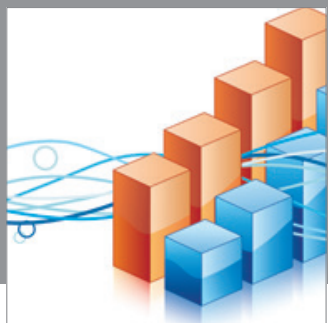

Advances in

Operations Research

mansans

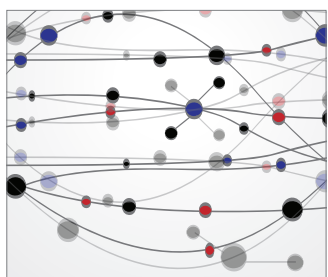

The Scientific World Journal
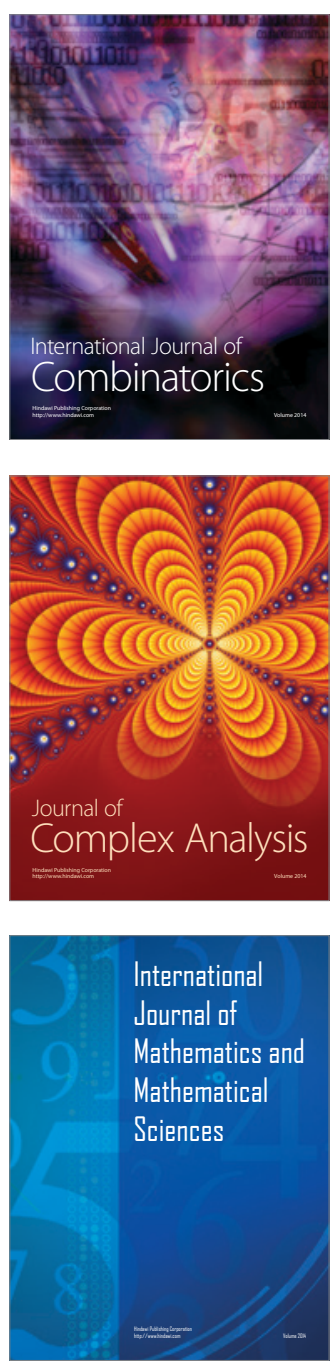
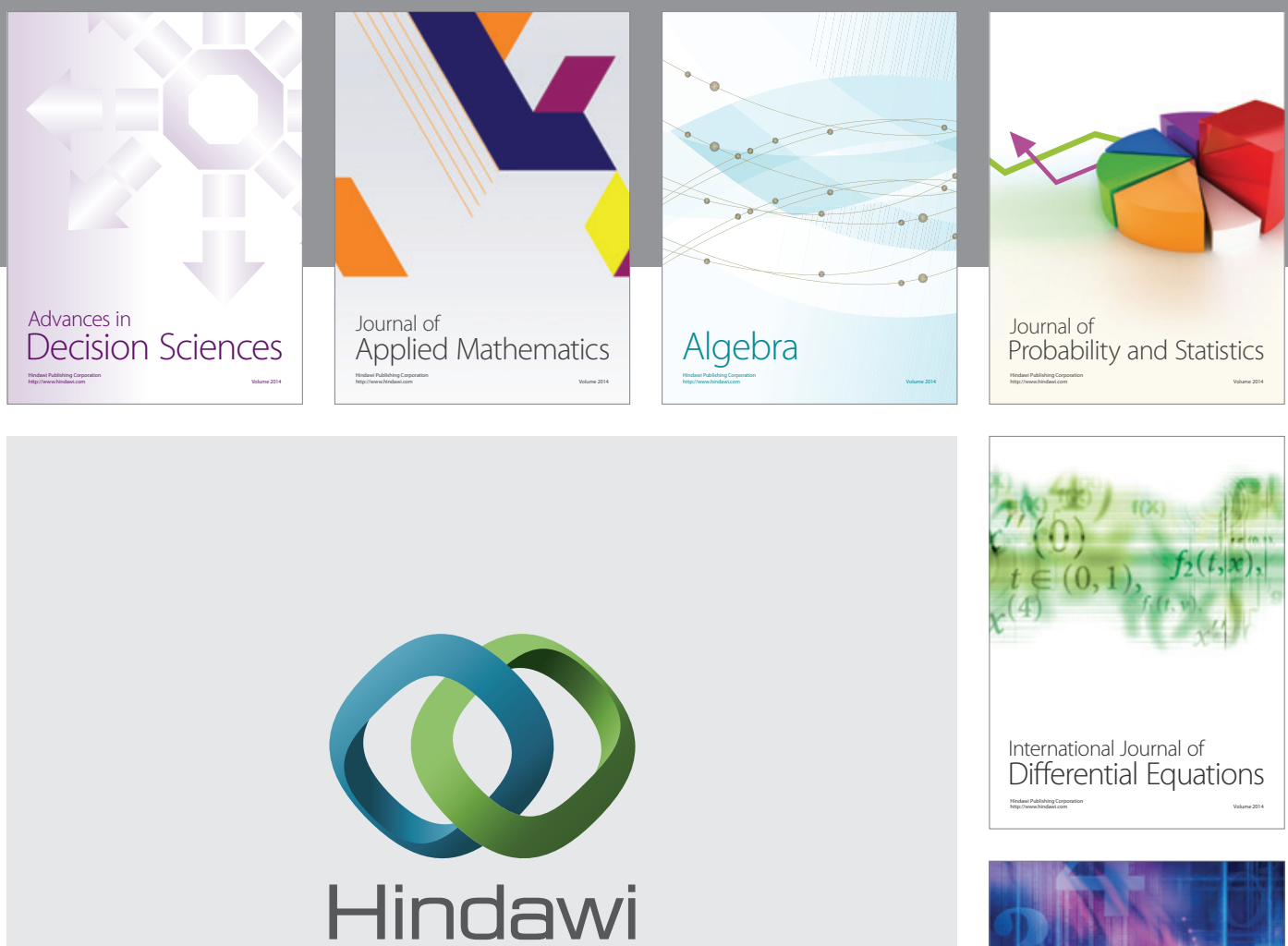

Submit your manuscripts at http://www.hindawi.com
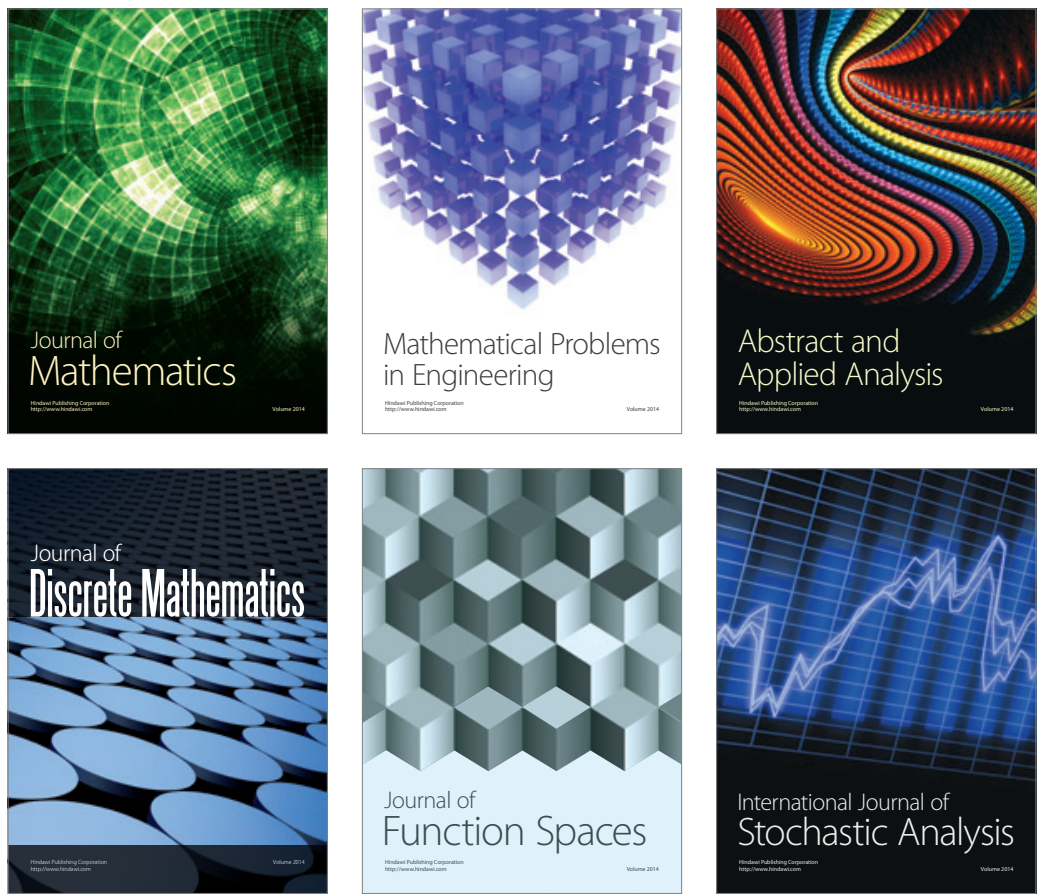

Journal of

Function Spaces

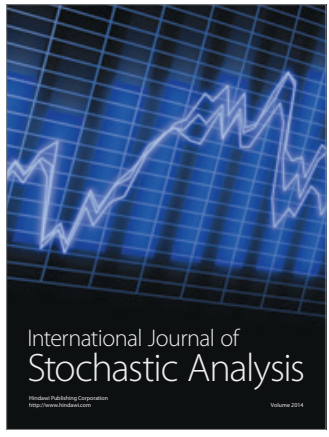

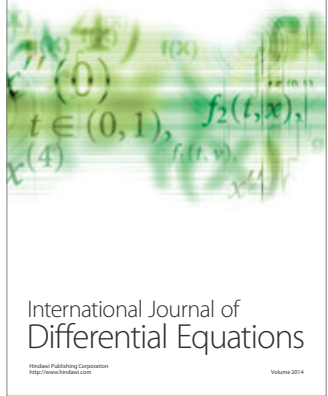
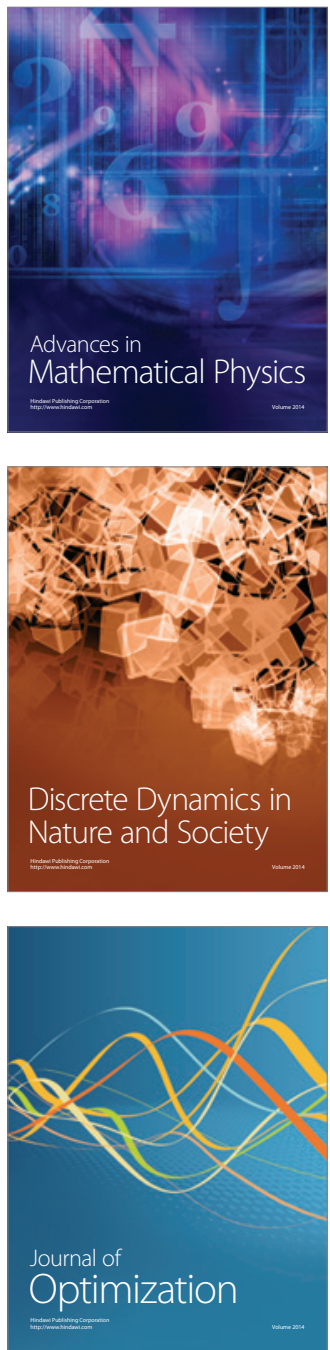